\title{
First detection of cold dust in the northern shell of NGC 5128 (Centaurus $A)^{\star}$
}

\author{
M. Stickel ${ }^{1}$, J. M. van der Hulst ${ }^{2}$, J. H. van Gorkom ${ }^{3}$, D. Schiminovich ${ }^{4}$, and C. L. Carilli ${ }^{5}$ \\ ${ }^{1}$ Max - Planck - Institut für Astronomie, Königstuhl 17, 69117 Heidelberg, Germany \\ 2 Kapteyn Astronomical Institute, Postbus 800, 9700 AV Groningen, The Netherlands \\ 3 Department of Astronomy, Columbia University, New York, NY 10027, USA \\ ${ }^{4}$ Division of Physics, Mathematics, and Astronomy, California Institute of Technology, MS 405-47, Pasadena, \\ CA 91125, USA \\ 5 National Radio Astronomy Observatory, PO Box 0, Socorro, NM 87801, USA
}

Received 12 September 2003 / Accepted 3 November 2003

\begin{abstract}
Deep far-infrared (FIR) imaging data obtained with ISOPHOT at $90 \mu \mathrm{m}, 150 \mu \mathrm{m}$, and $200 \mu \mathrm{m}$ detected the thermal emission from cold dust in the northern shell region of NGC 5128 (Centaurus A), where previously neutral hydrogen and molecular gas has been found. A somewhat extended FIR emission region is present in both the $150 \mu \mathrm{m}$ and $200 \mu \mathrm{m}$ map, while only an upper flux limit could be derived from the $90 \mu \mathrm{m}$ data. The FIR spectral energy distribution can be reconciled with a modified blackbody spectrum with very cold dust color temperatures and emissivity indices in the range $13 \mathrm{~K}<T_{\text {Dust }}<15.5 \mathrm{~K}$ and $2>\beta>1$, respectively, where the data favor the low temperature end. A representative value for the associated dust mass is $M_{\text {Dust }} \approx 7 \times 10^{4} M_{\odot}$, which together with the HI gas mass gives a gas-to-dust ratio of $\approx 300$, close the average values of normal inactive spiral galaxies. This value, in conjunction with the atomic to molecular gas mass ratio typical for a spiral galaxy, indicates that the interstellar medium (ISM) from the inner part of a captured disk galaxy is likely the origin of the outlying gas and dust. These observations are in agreement with recent theoretical considerations that in galaxy interactions leading to stellar shell structures the less dissipative clumpy component of the ISM from the captured galaxy can lead to gaseous shells. Alternatively, the outlying gas and dust could be a rotating ring structure resulting from an interaction or even late infall of tidal material of a merger in the distant past. With all three components (atomic gas, molecular gas, dust) of the ISM present in the northern shell region, local star formation may account for the chains of young blue stars surrounding the region to the east and north. The dust cloud may also be involved in the disruption of the large scale radio jet before entering the brighter region of the northern radio lobe.
\end{abstract}

Key words. galaxies: individual: NGC 5128 - galaxies: elliptical \& lenticular, cD - galaxies: intergalactic medium - infrared: general - infrared: galaxies

\section{Introduction}

Gravitational interaction is a major process rearranging the stars and the interstellar medium (ISM) of the involved progenitor galaxies. The more violently and supposedly young cases of gravitational interactions are easily discernible by their optically highly disturbed appearance, particularly in the case of disk galaxies. The presumably older, more settled, and perhaps virialized later stages can be ascribed to the rarer optical features of shells and polar rings, which consist mostly of stars.

The fate of the ISM can be traced by the structure of the atomic hydrogen (HI) gas, which is relatively easy to observe

Send offprint requests to: M. Stickel,

e-mail: stickel@mpia-hd.mpg.de

* Based on observations with ISO, an ESA project with instruments funded by ESA Member States (especially the PI countries: France, Germany, The Netherlands and the UK) and with the participation of ISAS and NASA. and often found far off the galaxy centers in irregular structures, even when the optical images no longer show obvious signs of interactions (Hibbard 2000; Hibbard et al. 2001). However, the HI gas in the progenitor galaxies is usually much more extended than the stars, the molecular gas, and the dust. Its distribution in the interacting system is therefore not necessarily representative of these other components of the ISM. Moreover, a detection of molecular gas and dust in the interacting system provides evidence from where the observed ISM has been torn away and thereby can constrain models of the interaction process.

While molecular hydrogen as traced by the $\mathrm{CO}$ emission is now regularly found in the inner regions of disk galaxies (Helfer et al. 2003, and references therein), the detection of molecular gas far from the disk is rather rare. Besides its occurrence in Tidal Dwarf Galaxies, possibly gravitationally bound systems in the tails of violently interacting galaxies where it is likely condensing out of the HI (Braine et al. 2001), only a 
few additional cases with outlying molecular $\mathrm{CO}$ emission have been found (Braine et al. 2001; Aalto et al. 2001). Similarly, the FIR or sub-mm thermal emission of compact extra-nuclear dust structures is detected in strongly interacting systems such as NGC 4038/39 ("the Antennae", Haas et al. 2000) and HCG 92 ("Stephans Quintett", Sulentic et al. 2001), whereas in weakly interacting or less disturbed systems none has been reported so far. A possible exception might be a cold intergalactic dust cloud in the outskirts of the Virgo elliptical M86 (Stickel et al. 2003), which is likely undergoing tidal interactions during its infall into the Virgo cluster.

Any FIR emission from extra-nuclear dust associated with the atomic and molecular ISM components in a weakly interacting system is likely quite faint, even if galactic gas-todust ratios are assumed. Thus, a search for outlying dust from a captured system is promising only in nearby galaxies. A prime candidate in this respect is the radio galaxy NGC 5128 (Centaurus A), an optically highly disturbed system with a central dust lane, which is considered to result from at least one major merger of an elliptical system with a spiral galaxy. It is also particularly interesting because of its system of optical shells (Malin et al. 1983; Peng et al. 2002), which is possibly the result from one or even more earlier minor encounters. The morphology seen in deep optical images bears some resemblance to a polar ring galaxy (Richter et al. 1994) or even to a spheroid intersected by a large disk oriented along a northeast south-west direction (Malin 1978; Cannon 1981; Haynes et al. 1983; Peng et al. 2002). Other remarkable features include a highly complex radio structure (Morganti et al. 1999) and emission line regions roughly aligned with the radio jet (Blanco et al. 1975; Morganti et al. 1991; Graham 1998). A more detailed review of the current observational status of NGC 5128 can be found in Israel (1998), while schematic diagrams of the various optical, radio, and cold gas structures can be found in Dufour \& van den Bergh (1978), Morganti et al. (1999) and Charmandaris et al. (2000), respectively.

The detection of HI emission in several regions near the outer shells (Schiminovich et al. 1994) and the subsequent detection of molecular gas traced by $\mathrm{CO}$ emission in the two regions with the strongest $\mathrm{HI}$ emission (Charmandaris et al. 2000) indicated that the ISM in the tidal remnant has not yet completely settled in the center of the potential (cf. Weil \& Hernquist 1993). This is possibly due to a relatively high velocity of the encounter, as indicated by the rotation of the HI gas (Schiminovich et al. 1994) and the azimuthally distributed shell system (Peng et al. 2002). Alternatively, tidal material might be falling back onto the main galaxy long after the encounter, as suggested by Hibbard \& van Gorkom (1996) and recently confirmed by numerical simulations (Barnes 2002). The HI morphology and velocity field of NGC 5128 (Schiminovich et al. 1994) strikingly resembles a ring structure, similar to a few rare cases of HI rings (Barnes 1999; Ryan-Weber et al. 2003, and references therein), in which case the positional association with the stellar shells might only be a projection effect. In fact, locating the three HI emission regions (Schiminovich et al. 1994) in the optical image showing the interleaved shell system (Peng et al. 2002) reveals that only the northern S1 region lies outside the outermost shell whereas the other two are situated between shells. The CO detection in the northern shell region S1 was confirmed by Curran (2001), although a different interpretation in terms of an molecular outflow associated with the radio jet was suggested.

NGC 5128 had been the target of previous FIR studies based on IRAS data (Israel 1998, and references therein), as well as more recently, in the near-IR (Mirabel et al. 1999) and sub-mm (Leeuw et al. 2002). These studies were aimed at the investigation of the dust emission of the already optically visible dust disk in the central part of the disturbed galaxy. Up to now, no evidence has shown up of any localized offcenter FIR emission, particularly none at the position of the optical shells or associated with the outlying off-center HI and CO emission. In the case the IRAS data (Marston \& Dickens 1988; Eckart et al. 1990; Marston 1992; LeWinter et al. 1993), this negative result might not only be due to sensitivity but also due to the wavelength limit of $100 \mu \mathrm{m}$, which made it insensitive to a cold dust component with temperatures below $20 \mathrm{~K}$.

The ISOPHOT detector (Lemke et al. 1996; Lemke \& Klaas 1999) aboard the Infrared Space Observatory (ISO; Kessler et al. 1996) not only provided a higher sensitivity and a higher angular resolution, its increased wavelength coverage out to $200 \mu \mathrm{m}$ also allows the detection of cold dust with temperatures well below $20 \mathrm{~K}$. Such cold dust temperatures might be expected since there is no strong radiation field from a large number of young stars which could heat any outlying dust. NGC 5128 was therefore observed with ISOPHOT to search for extra-nuclear dust in the northern part of the stellar halo, particularly dust associated with extra-nuclear $\mathrm{HI}$ and $\mathrm{CO}$ in the northern shell S1, the optical filaments, and the radio jet.

\section{Observations and data reduction}

An area of $\approx 20^{\prime} \times 20^{\prime}$ north of NGC 5128 was imaged at $150 \mu \mathrm{m}$ and $200 \mu \mathrm{m}$ with the ISOPHOT (Lemke et al. 1996; Lemke \& Klaas 1999) in August 1996. The main body of the galaxy was placed at the southern edge of the maps to cover the northern shell region S1 (Charmandaris et al. 2000), where previously HI and CO had been detected (Schiminovich et al. 1994; Charmandaris et al. 2000). These long wavelength observations were carried out with the ISOPHOT C200 camera, a $2 \times 2$ pixel array of stressed Ge:Ga with a pixel size of 89.4 in the regular raster mapping mode. The total integration time for the two filters was $4500 \mathrm{~s}$. A somewhat larger region north of NGC 5128 was observed at $90 \mu \mathrm{m}$ with the C100 camera of ISOPHOT, a $3 \times 3$ pixel array with a pixel size of $43^{\prime \prime} .5$ in the chopped raster mapping mode. The full area was mosaiced in eight separate parts in July 1996 with a total integration time of $6600 \mathrm{~s}$. For all observations, calibration measurements with the on-board Fine Calibration Source (FCS) of ISOPHOT were taken before and after each (sub-)map to put the observed signals on an absolute level.

The signal derivation for each detector pixel and sky position made use of the full distribution of pairwise ramp readout differences instead of ramp slope fitting. This gives a much better rejection of cosmic ray hits, which is critical for the detection of faint FIR sources (Stickel et al. 2003). The signals were corrected for the dependence on ramp integration times to 
be consistent with calibration observations, dark-current subtracted, and finally flux calibrated with ISOPHOT Interactive Analysis package PIA ${ }^{1}$ version 9.1 / Cal G version 6.0 (Gabriel et al. 1997). For the conversion of detector signals to fluxes, the average of the signals of the two FCS measurements accompanying each (sub-)map was used.

The flux calibrated data streams of the detector pixels of all observations showed significant differences in the overall sky levels levels of up to $30 \%$, most likely coming from inappropriately corrected pixel-to-pixel sensitivities (flatfield), which moreover appeared to be time-dependent. If uncorrected, this would lead to quite severe striping and checker board patterns in the final maps, thereby degrading the limit for detecting faint structures significantly. Therefore, the data streams were smoothed with robust filtering techniques, and each individual data stream rescaled to the common mean, while any residual time trend was removed with robust low-order polynomial fits. However, due to the very high contrast between the FIR bright central disk and the very low FIR emission in the outer stellar halo of NGC 5128, an acceptable flattening of the whole observed region could not be achieved. Since the central parts of the disk region is in any case saturated in all three filters, the flat-fielding has therefore been optimized for the outer stellar halo region of NGC 5128 .

The ISOPHOT raster mapping observations contain an inherent redundancy such that a given sky position is observed with several pixels, and additionally, in the case of the $90 \mu \mathrm{m}$ chopped measurement with the $\mathrm{C} 100$ detector, during several chopper sweeps. For the longer wavelengths C200 observations at $150 \mu \mathrm{m}$ and $200 \mu \mathrm{m}$, this redundancy was utilized by producing for each detector pixel a full map with a common center using the Drizzle mapping method (Hook \& Fruchter 1997) within IRAF ${ }^{2}$. Similarly, full maps with a common center were created for each pixel and chopper throw from the $90 \mu \mathrm{m}$ data streams, again with the Drizzle mapping method. These individual maps were subsequently stacked, and a final map created from the stack. In the case of the C200 detector with only 4 pixels, a robust biweighted mean was applied to each pixel in the stack. For the $90 \mu \mathrm{m}$ map, the stack consists of a total of 117 full images, which were averaged after applying a minmax outlier rejection scheme. This stack-averaging reduction scheme is similar to standard near-infrared data processing and has been shown to suppress quite effectively small scale detector variability and cosmic ray hits, leading to significantly lower detection limits (Stickel et al. 2003).

For cross-checking the obtained results, full maps have additionally been created in a single drizzling step from the pairwise as well as the standard linear ramp fitted data streams. Furthermore, the total integration time per sky position was divided into two halves and the pairwise signals from each

\footnotetext{
1 The ISOPHOT data presented in this paper were reduced using PIA, which is a joint development by the ESA Astrophysics Division and the ISOPHOT Consortium. The ISOPHOT Consortium is led by the Max-Planck-Institute für Astronomie, Heidelberg.

${ }^{2}$ IRAF is distributed by the National Optical Astronomy Observatories, which are operated by the Association of Universities for Research in Astronomy, Inc., under cooperative agreement with the National Science Foundation.
}

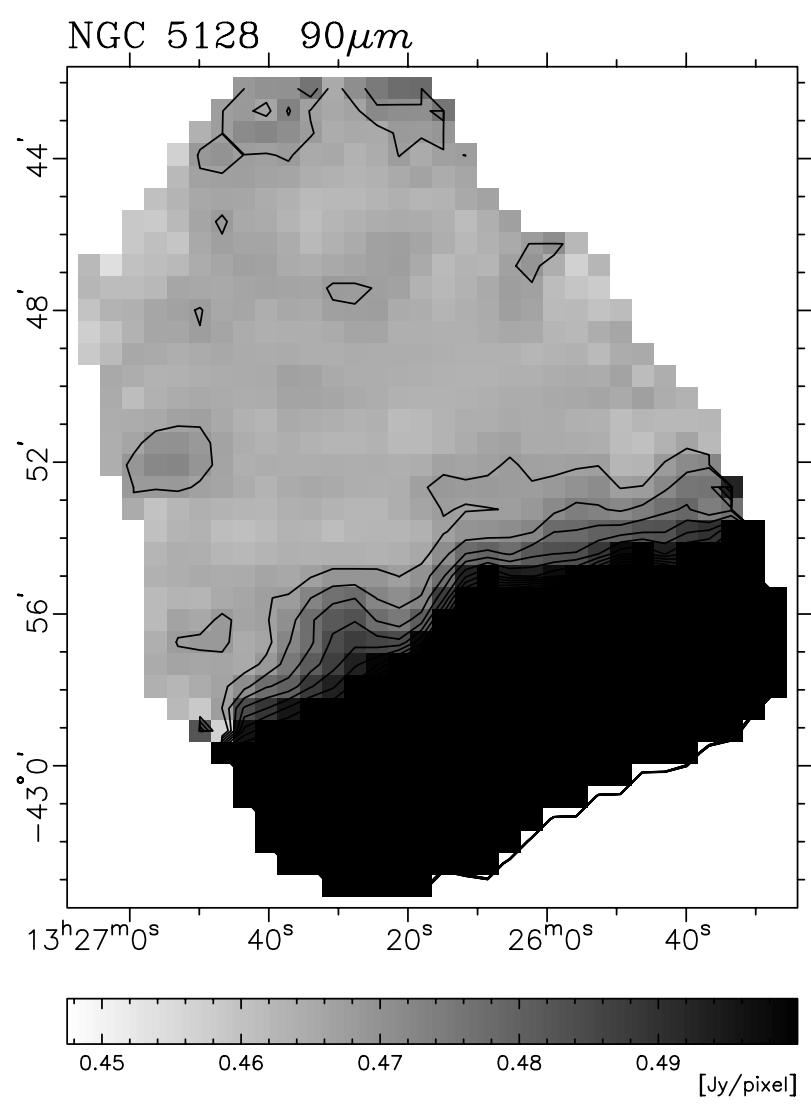

Fig. 1. Gray-scale representation with overlaid isocontours of the ISOPHOT $90 \mu \mathrm{m}$ map of the region north of NGC 5128. The large dark blob at the bottom is the FIR emission of the central dusty disk region of NGC 5128 itself. Only a few very low level structures near the edges of the mapped region are apparent. Map pixel size is $35^{\prime \prime}$.

part treated as completely separate measurements, which were again fully processed to the complete maps. Except for an increased noise due to the less robust signal derivation, these four additional sets of maps showed results consistent with the above described pairwise stack maps, thereby providing an independent confirmation of the features described below.

\section{Results}

The final $90 \mu \mathrm{m}$ (Fig. 1), $150 \mu \mathrm{m}$ (Fig. 2) and $200 \mu \mathrm{m}$ (Fig. 3) maps are dominated by FIR emission of the central dusty disk of NGC 5128. The galaxy center is saturated in all three bands, although this is not obvious from the displayed images. Unfortunately, this precludes the derivation of integral fluxes for the central part of the galaxy. As a consequence of the high fluxes, long term drifts were apparent in the one-dimensional data streams, which could not be removed completely by the above described flat-fielding process. Any structures directly adjacent to the central dust disk must therefore be considered unreliable.

In the $90 \mu \mathrm{m}$ map, no FIR emission further away from the central disk is seen, particularly not at the position of the HI (Schiminovich et al. 1994) and CO (Charmandaris et al. 2000) in the northern shell S1, nor is there anything discernible at the position of the northern large scale radio jet 


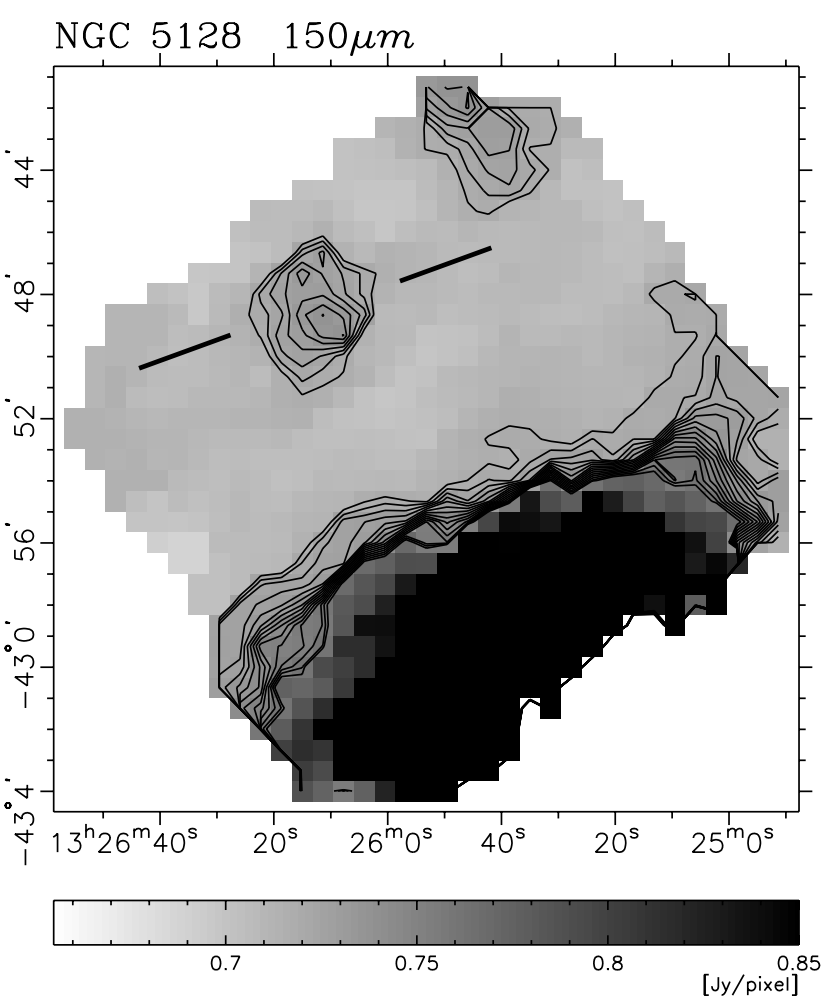

Fig. 2. Gray-scale representation with overlaid isocontours of the ISOPHOT $150 \mu \mathrm{m}$ map. In addition to the strong FIR emission of the central region of NGC 5128 near the lower edge, a resolved emission region $\approx 15^{\prime}$ northeast of NGC 5128 (marked with a bar) can clearly be seen. Map pixel size is $40^{\prime \prime}$.

(Morganti et al. 1999) or the ionized diffuse clouds and filaments (Graham 1998). A few very low level structures lying close to the edges of the map are not significant, because their appearance is strongly dependent on the on the data set used (see above) to create the map. They are due to detector noise and residual baseline variations not completely removed by the flat field correction.

In contrast, the $150 \mu \mathrm{m}$ (Fig. 2) and $200 \mu \mathrm{m}$ (Fig. 3) maps show in addition to the bright FIR source due to the dusty central disk also a somewhat extended FIR source $\approx 15^{\prime}$ northeast of NGC 5128. Its peak emission is at the same position at both wavelengths $\left(\alpha_{2000}: 13^{\mathrm{h}} 26^{\mathrm{m}} 16^{\mathrm{s}}, \delta_{2000}:-42^{\circ} 48^{\prime} 35^{\prime \prime}\right)$, which in turn is closely coincident with the maximum of the HI (Schiminovich et al. 1994) and CO (Charmandaris et al. 2000) emission in the northern shell S1. The positional offset of the peak of the FIR emission $\left(\approx 1^{\prime}\right.$ south) from the $\mathrm{HI}$ and $\mathrm{CO}$ emission, the somewhat brighter southern end as well as the apparently faint curved extension of the FIR emission present in both maps, which in fact bears some resemblance to the curved HI morphology (Schiminovich et al. 1994), can not necessarily be taken as evidence for a strongly asymmetric FIR emission region, because these features could be a result of the asymmetric sampling of a compact dust cloud with the large $\left(1.5^{\prime}\right)$ ISOPHOT C200 pixels. However, it should be emphasized that the FIR emission in both maps lies away from the map border and could therefore not be due to edge effects, which result from the reduced redundancy and incomplete coverage of a

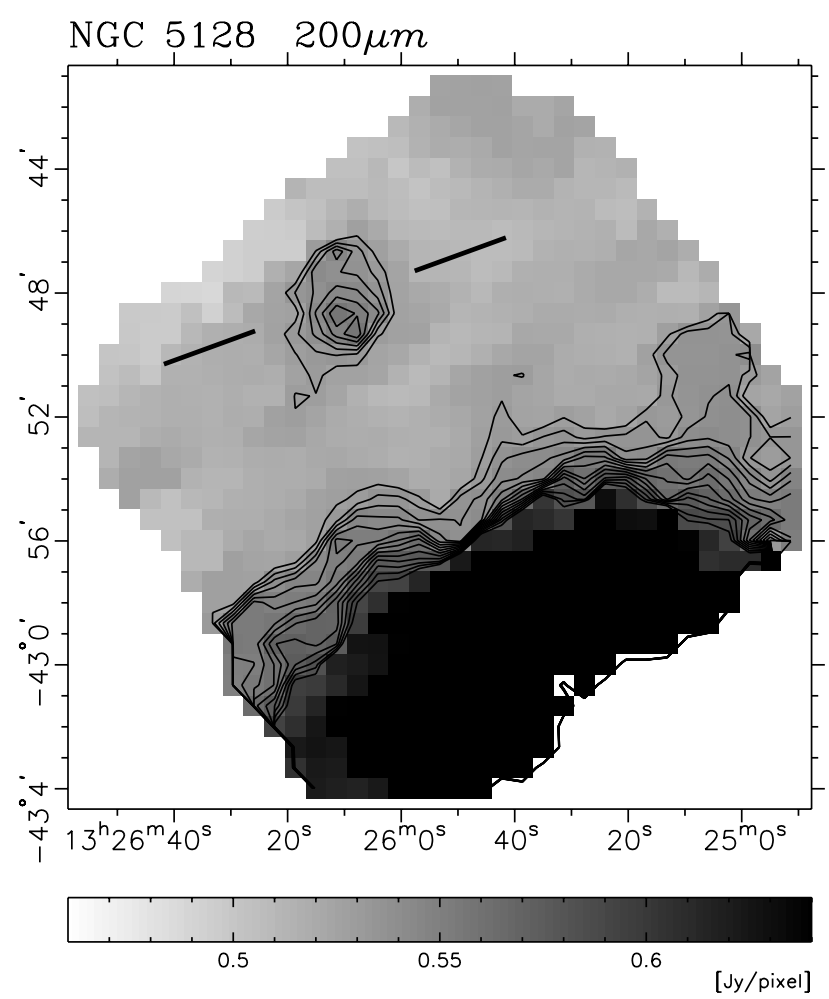

Fig. 3. Same as Fig. 2, but for the $200 \mu \mathrm{m}$ band.

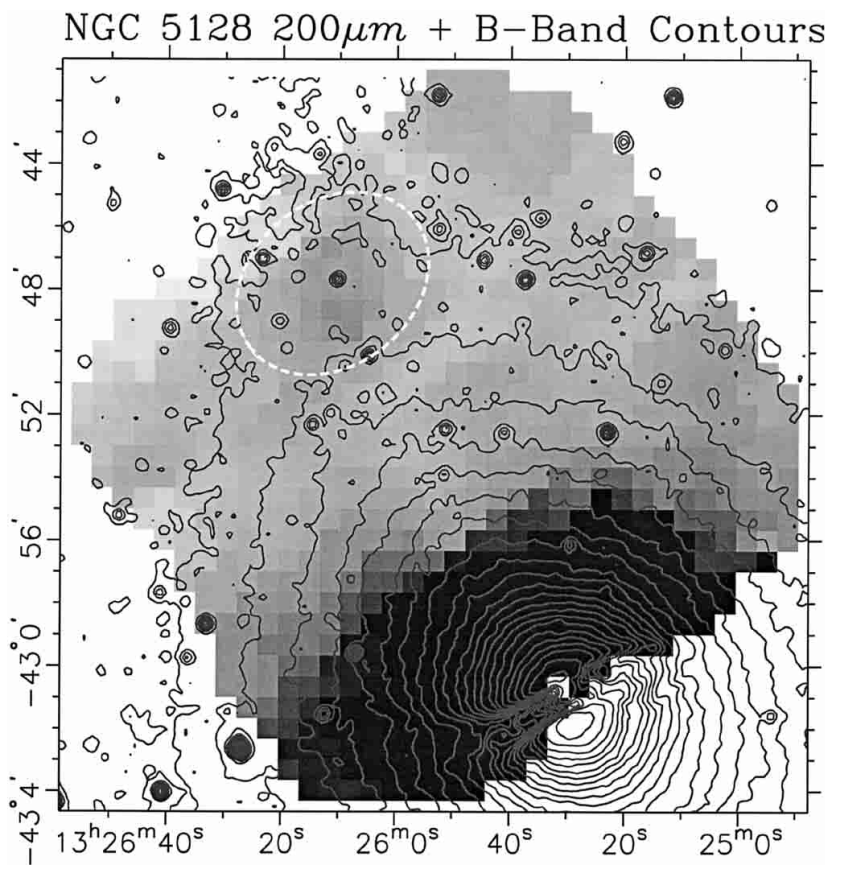

Fig. 4. Gray-scale representation of the ISOPHOT $200 \mu \mathrm{m}$ map with overlaid isocontours of the DSS-2 $B$-band image. The outlying FIR emission region is indicated by the white ellipse.

raster scan. Additionally, each of the $150 \mu \mathrm{m}$ and $200 \mu \mathrm{m}$ maps made from the other above described data streams showed the same FIR emission region, albeit with minor differences in its level and structure.

The combined $200 \mu \mathrm{m} /$ DSS-2 $B$-band image (Fig. 4) shows that the off-center FIR emission lies at the position 


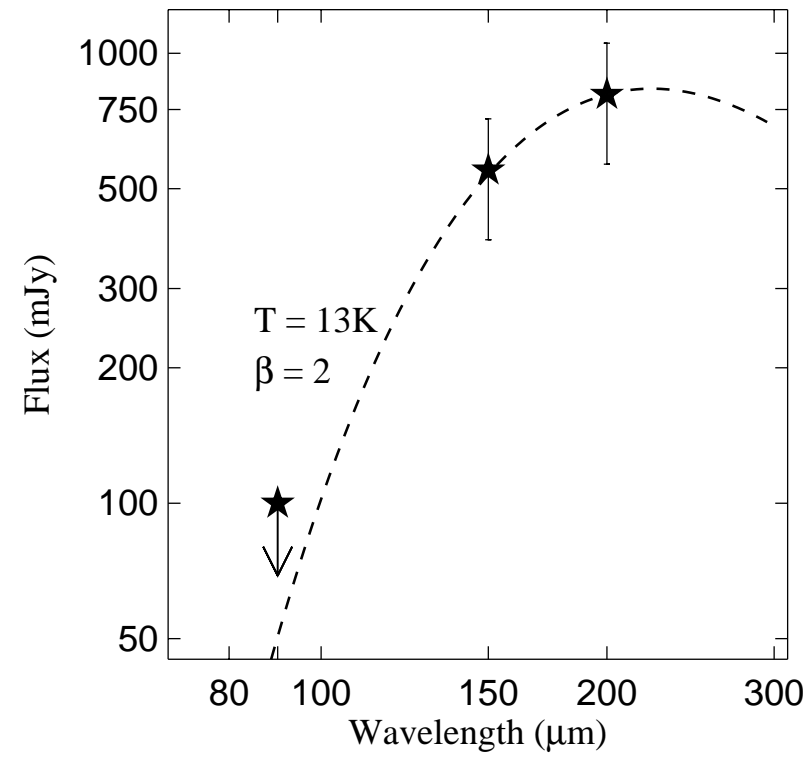

Fig. 5. FIR spectrum of the off-center source at the position of the $\mathrm{HI} / \mathrm{CO}$ detection in NGC 5128. 30\% error bars are indicated for the ISOPHOT detections at $150 \mu \mathrm{m}$ and $200 \mu \mathrm{m}$. The dashed curve shows a modified blackbody spectrum with temperature $T_{\text {Dust }}=13 \mathrm{~K}$ and emissivity $\beta=2$.

where the faint optical extensions seen in deep images (Malin 1978; Cannon 1981; Haynes et al. 1983) start, as indicated by the departure of the optical isophotes from elliptical symmetry. Remarkably, the diffuse $\mathrm{H}_{\alpha}$ emission (Fig. 1 of Blanco et al. 1975) has also a strong depression there.

Other known features of NGC 5128, namely dust patches seen in the optical (Dufour \& van den Bergh 1978), the arc of young blue stars north-west of the central dust disk (Peng et al. 2002; Karovska et al. 2002), the northern inner radio jet and lobe (Burns et al. 1983), and the inner gaseous filaments (Blanco et al. 1975; Morganti et al. 1991) lie in all three maps too close to the very bright central dust disk to be discernible, even if compact FIR emission up to a few Jy would have been present.

The integrated FIR fluxes of the northern emission region have been measured with simple aperture photometry. From the total flux inside a rectangular box containing the FIR emission, various background boxes were subtracted to give the net flux, while the variations of the differences gave an indication of the accuracy. In the $90 \mu \mathrm{m}$ map, twice the flux of the brightest blob near the eastern border was considered as the absolute minimum of any FIR emission, which would have been considered a detection if it were at the position of the FIR emission at $150 \mu \mathrm{m}$ and $200 \mu \mathrm{m}$ and the $\mathrm{HI} / \mathrm{CO}$ detections. The resulting fluxes are $550 \mathrm{mJy}$ at $150 \mu \mathrm{m}, 810 \mathrm{mJy}$ at $200 \mu \mathrm{m}$, while at $90 \mu \mathrm{m}$ a conservative upper limit for any FIR emission within a similar region is $100 \mathrm{mJy}$. Uncertainties in the $150 \mu \mathrm{m}$ and $200 \mu$ m fluxes are $\approx 30 \%$, as derived from several combinations of the source and different background regions.

The spectral energy distribution (SED, Fig. 5) shows that the FIR emission is strongly rising from $90 \mu \mathrm{m}$ to $150 \mu \mathrm{m}$, and is further increasing to $200 \mu \mathrm{m}$. Apparently, its peak is not covered by observed wavelength range and likely lies beyond $200 \mu \mathrm{m}$. The steep flux increase towards longer FIR wavelengths is indicative of the thermal emission of a cold dust component. The dust emission is usually represented by a modified blackbody (Planck) function

$F_{v} \propto v^{\beta} B_{v}\left(T_{\text {Dust }}\right)$

with a dust color temperature $T_{\text {Dust }}$ and an emissivity index $\beta$. The measured fluxes at $150 \mu \mathrm{m}$ and $200 \mu \mathrm{m}$ as well as the upper limit at $90 \mu \mathrm{m}$ do not strongly constrain the values for the dust color temperature and the emissivity index. For emissivity indices in the range $2>\beta>1$, they can be reconciled with dust color temperatures in the range $13 \mathrm{~K}<T_{\text {Dust }}<15.5 \mathrm{~K}$. However, with increasing dust color temperature and decreasing emissivity index, the SED becomes increasingly flatter towards shorter wavelengths, and the combination $\beta=1$ and $T_{\text {Dust }}=15.5 \mathrm{~K}$ gives a $90 \mu \mathrm{m}$ flux hardly below the conservative detection limit indicated in Fig. 5. The detections at $150 \mu \mathrm{m}$ and $200 \mu \mathrm{m}$ together with the upper limit at $90 \mu \mathrm{m}$ thus favor a dust temperature towards the lower and an emissivity index towards the upper end of the possible range. Moreover, if a inverse temperature dependence of the emissivity index similar to that found in the Milky Way (Dupac et al. 2003) holds also for the outlying dust in NGC 5128, then only $\beta \approx 2$ with $T_{\text {Dust }} \approx 13 \mathrm{~K}$ is acceptable. Given the uncertainties in the measured fluxes at $150 \mu \mathrm{m}$ and $200 \mu \mathrm{m}$, the dust color temperature might be even lower than $13 \mathrm{~K}$.

The dust mass associated with the FIR emission can be estimated from

$$
M_{\text {Dust }}=D^{2} F_{v}\left[\kappa_{\lambda} B_{v}\left(T_{\text {Dust }}\right)\right]^{-1}
$$

(Hildebrand 1983; Draine 1990), where $F_{v}$ is the flux density, $D$ the distance, $\kappa_{\lambda}$ the dust opacity, and $T_{\text {Dust }}$ the dust color temperature. Dust opacities are rather uncertain and a representative value of $3 \mathrm{~m}^{2} / \mathrm{kg}$ for $200 \mu \mathrm{m}$ is used, which lies in the middle of the range of currently considered values (Draine 1990). With a distance to NGC 5128 of 3.4 Mpc (Israel 1998), the derived dust mass for dust color temperatures $13 \mathrm{~K}<T_{\text {Dust }}<$ $15.5 \mathrm{~K}$ lies in the range $8 \times 10^{4} M_{\odot} \gtrsim M_{\text {Dust }} \gtrsim 3.2 \times 10^{4} M_{\odot}$.

\section{Discussion}

With the detection of the FIR emission from cold dust at the position of previously detected $\mathrm{HI}$ and $\mathrm{CO}$ in the northern shell S1 of NGC 5128, all three components of the ISM, atomic and molecular gas as well as dust, have now for the first time been seen in an outer shell structure of an early type galaxy. As it is obvious from the spectral energy distribution (Fig. 5), the newly discovered extra-nuclear dust is rather cold having a color temperature below $14 \mathrm{~K}$, which is in fact significantly colder than the dust color temperature of the Milky Way (Sodroski et al. 1994) and of other normal inactive spiral galaxies (Stickel et al. 2000), but similar to cold interstellar clouds in the Milky Way (Ristorcelli et al. 1998; Tóth et al. 2000). This low temperature together with the rather small inferred dust mass leads to very low ( $\$ 100 \mathrm{mJy}$ ) FIR fluxes at wavelengths up to $100 \mu \mathrm{m}$, which in turn precluded an earlier detection with IRAS. 
FIR spectra and dust temperatures for dispersed ISM dust out to large radii from the galaxy center have been computed for ellipticals by Temi et al. (2003). This model includes heating by star light as well as by electron - grain collisions in a hot $\mathrm{X}$-ray emitting component. An exemplary result of the grain temperature as a function of distance was given for NGC 4636 (Fig. 2 in Temi et al. 2003), which has a total mass and effective radius quite similar to that of NGC 5128 (Israel 1998). It shows that large dust grains can actually attain very low temperatures $\left(T_{\text {Dust }}<15 \mathrm{~K}\right)$ in the outer regions, only heated by the ambient starlight. This is in accord with the low temperature of the dust in the northern shell S1 of NGC 5128 (Fig. 5), which can be taken as evidence that neither the surrounding chains of young stars nor the radio jet (see below) is significantly contributing to the heating of at least the large dust grains. Unfortunately, no deep MIR/FIR data between $20 \mu \mathrm{m}$ and $90 \mu \mathrm{m}$ are available for the northern outskirts of NGC 5128 to check the presence of MIR/FIR emission from smaller grains. They are expected to have significantly higher temperatures, partly because electrongrain collisions become important (Temi et al. 2003). The absence of thermal emission from these smaller grains to very low detection limits would indicate that they might have already been destroyed by sputtering (Dwek et al. 1990; Tielens et al. 1994; Dwek et al. 1996), leaving preferentially behind the larger grains. The grain sputtering time in turn can be used to set a lower limit for the lifetime of the dust cloud, which for an external merger origin of the dust might give an indication when this event had happened.

For the northern shell S1, Charmandaris et al. (2000) give a HI gas mass of $\approx 2.1 \times 10^{7} M_{\odot}$, and an only slightly smaller amount of molecular gas. Taking $\approx 7 \times 10^{4} M_{\odot}$ as a representative value for the dust mass gives a $\mathrm{HI}$ gas-to-dust ratio of $\approx 300$. Since the total gas HI mass is distributed over a much larger area than the detected FIR emission (Schiminovich et al. 1994), the HI gas-to-dust ratio of the inner region of the northern shell is likely smaller. Given the uncertainties in the dust parameters, this value is remarkably close to the canonical value of the Milky Way ( $\approx 160$, Sodroski et al. 1994), and even closer to the median value of $\approx 250$ for a large number of inactive spiral galaxies (Stickel et al. 2000). Similarly, the ratio of the neutral to molecular gas mass of $\approx 1.25$ is also representative for spiral galaxies (Charmandaris et al. 2000).

There is no report of a large cluster of young stars or a large evolved star formation region associated with the northern shell region $\mathrm{S} 1$, which makes a creation of dust from the late phases of the stellar evolution unlikely. Rather, the observed properties of the extra-nuclear gas and dust strongly suggest an external origin, most likely coming from the normal ISM of a gas rich progenitor galaxy, while a gas poor elliptical or spheroidal galaxy and a low metallicity dwarf or low surface brightness galaxy appears to be ruled out.

The presence of dust in the northern shell already excludes the outer HI rich envelope far beyond the stellar disk of a spiral galaxy as the origin of the gaseous shell component, as suggested by Carter (1999). CO has been found to be a good tracer not only for molecular hydrogen gas but also for dust (Neininger et al. 1998, and references therein). Its confinement to the stellar disk region (Regan et al. 2001; Helfer et al. 2003, and references therein) provides strong evidence for the general absence of dust at very large radii, although there have been a few hints that dust can indeed reside outside stellar disks (Nelson et al. 1998; Alton et al. 1998; Melo et al. 2002). The approximately normal properties of the shell material then indicates that the ISM of the inner part of a spiral galaxy is its likely origin.

The detection of dust is also in agreement with models for the shell formation (Combes \& Charmandaris 2000; Charmandaris \& Combes 2000), where the ISM of a merging companion is not only included as a smoothly distributed, homogeneous dissipative component, but also contains a clumpy component of small and dense clouds with a very low dissipation, which in turn will survive and be present in the shell structures along with the stellar component. The consistent picture of an external origin of the outlying gas and dust also makes an collimated outflow of molecular gas from the central regions along the jets (Curran 2001) for the origin of the CO emission in the northern shell S1 less likely, although some outflow of ISM components from the central actively star forming region of NGC 5128 in form of a galactic wind is to be expected.

Since dust is a necessary requisite for molecular gas formation, its presence in the northern shell $\mathrm{S} 1$ can be taken as evidence that at least a fraction of the molecular gas might have been created in situ from the neutral gas, rather than brought in from the captured system. This situation would then be similar to a few tidal dwarf galaxies, where the formation of molecular gas out of HI and dust has been proposed (Braine et al. 2001). The presence of atomic and molecular gas together with dust in a compact cold region is reminiscent of Giant Molecular Clouds in the Milky Way, which often harbor the sites of star formation. There is currently no evidence for large scale star formation going on at the center of the northern gas and dust cloud. However, there is a chain of young blue stars not only along the eastern edge of the cloud (Rejkuba et al. 2001, 2002) adjacent to the emission line region likely excited by the radio jet (Morganti et al. 1991; Graham 1998), but remarkably also along its northern edge (Fassett \& Graham 2000). This can be interpreted as recent star formation at the surface of the cloud, which initially might have been triggered by its interaction with the jet, but apparently has now spread even to the far side of the supposed jet-cloud interface. Even earlier phases of the stellar evolution are characterized by compact cores with gas and dust temperatures well below $15 \mathrm{~K}$, which usually show up as intensity peaks in cold gas and dust clouds at the longest FIR and sub-mm wavelengths. Whether such pre-stellar cores are present and star formation might eventually independently start within the gas and dust cloud can be tested by high resolution sub-mm mapping.

A remarkable feature has shown up in recent $20 \mathrm{~cm}$ mapping of the brighter northern radio lobe region, namely a large scale jet connecting the inner radio lobe and the much more diffuse radio emitting region of the northern middle lobe (Morganti et al. 1999). As already noted by Morganti et al. (1999), the position where the confined jet emission becomes diffuse is positionally coincident with the $\mathrm{HI}$ and $\mathrm{CO}$ emission. The newly detected dust at the same position can be taken as 
supporting evidence that there is indeed some kind of dynamical interaction going on which disrupts the collimated jet.

Although a few other cases of shell galaxies with associated HI emission are known (Schiminovich et al. 1995; Petric et al. 1997; Balcells et al. 2001; Schiminovich 2001), no other clear case of $\mathrm{CO}$ emission associated with the $\mathrm{HI}$ is currently published. An uncertain case is NGC 3656, where Charmandaris $\&$ Combes (2000) note a possible detection of the southern shell. Unfortunately, this particular galaxy has not been observed with ISO at FIR wavelengths. A cross-correlation of the FIR mapping observations in the ISO data archive with the lists of shell galaxies from Malin \& Carter (1980), Wilkinson et al. (1987), and Thronson et al. (1989) revealed that there are several additional shell galaxies with useful mapping data at wavelengths beyond $60 \mu \mathrm{m}$. Among them are the two well known cases NGC 474 and Arp 230, which have in addition to stellar shells also a highly structured HI morphology. Again, no $\mathrm{CO}$ has been detected associated with the optical shells in these galaxies (Combes et al., priv. commun.). The ISOPHOT observations showed in both cases no outlying compact dust structures, and did detect the central FIR emission only from Arp 230 but not from NGC 474. However, the less well known object NGC 5813 from the list of Thronson et al. (1989) clearly does show two outlying compact FIR emission regions not associated with anything obvious in the optical DSS- $2 R$ band image of the galaxy, although its center is only very weakly detected. NGC 5813 is a rather unconspicious E1 elliptical, for which a merger origin has been suggested by Kormendy (1984) on the basis of a non-isothermal core-witin-a-core structure, but has not yet been investigated in detail with HI observations. A detailed description of these archival FIR data from the ISO database will be given elsewhere (Stickel et al., in preparation). Since these three objects and all other shell galaxies with FIR mapping data are at much larger distances than NGC 5128, a similarly small amount of dust can currently not be detected. For the time being, the detection of dust in the northern shell system of NGC 5128 is therefore unique.

Acknowledgements. The development and operation of ISOPHOT were supported by MPIA and funds from Deutsches Zentrum für Luftund Raumfahrt (DLR, formerly DARA). The ISOPHOT Data Centre at MPIA is supported by Deutsches Zentrum für Luft- und Raumfahrt (DLR) with funds of Bundesministerium für Bildung und Forschung, grant. no. 50 QI0201.

This research has made use of NASA's Astrophysics Data System Abstract Service, the Simbad Database, operated at CDS, Strasbourg, France, and data from the Infrared Processing and Analysis Center (IPAC) and the NASA/IPAC Extragalactic Database (NED), which are operated by the Jet Propulsion Laboratory, California Institute of Technology, under contract with the National Aeronautics and Space Administration.

Based on photographic data obtained using The UK Schmidt Telescope. The UK Schmidt Telescope was operated by the Royal Observatory Edinburgh, with funding from the UK Science and Engineering Research Council, until 1988 June, and thereafter by the Anglo-Australian Observatory. Original plate material is copyright (c) the Royal Observatory Edinburgh and the Anglo-Australian Observatory. The plates were processed into the present compressed digital form with their permission. The Digitized Sky Survey was produced at the Space Telescope Science Institute under US Government grant NAG W-2166.

\section{References}

Aalto, S., Hüttemeister, S., \& Polatidis, A. G. 2001, A\&A, 372, L29 Alton, P. B., Trewhella, M., Davies, J. J., et al. 1998, A\&A, 335, 807 Balcells, M., van Gorkom, J. H., Sancisi, R., \& del Burgo, C. 2001, AJ, 122, 1758

Barnes, D. G. 1999, PASA, 16, 77

Barnes, J. 2002, MNRAS, 333, 481

Braine, J., Duc, P.-A., Lisenfeld, U., et al. 2001, A\&A, 378, 51

Blanco, V. M., Graham, J. A., Lasker, B. M., \& Osmer, P. S. 1978, ApJ, 198, 63

Burns, J. O., Feigelson, E. D., \& Schreier, E. J. 1983, ApJ, 273, 128

Cannon, R. D. 1981, in Optical Jets in Galaxies, ed. B. Battrick, \& J. Mort, ESA-SP 162, 45

Carter, D. 1999, in Galaxy Interactions at Low and High Redshifts, Proc. IAU Symp., 186, ed. J. E. Barnes, \& D. B. Sanders (Dordrecht: Kluwer), 165

Charmandaris, V., Combes, F., \& van der Hulst, J. M. 2000, A\&A, 356, L1

Charmandaris, V., \& Combes, F. 2000, in Small Galaxy Groups, ed. M. Valtonen, \& C. Flynn, ASP Conf. Ser., 209, 273

Combes, F., \& Charmandaris, V. 2000, in Dynamics of Galaxies: from the Early Universe to the Present, ed. F. Combes, G. A. Mamon, \& V. Charmandaris, ASP Conf. Ser., 197, 339

Curran, S. J. 2001, A\&A, 376, 402

Draine, B. T. 1990, in The Interstellar Medium in Galaxies, ed. H. A. Thronson Jr., \& J. M. Schull (Dordrecht: Kluwer), 483

Dufour, R. J., \& van den Bergh, S. 1978, ApJ, 226, 73

Dupac, X., Bernard, J.-P., Boudet, et al. 2003, A\&A, 404, L11

Dwek, E., Rephaeli, Y., \& Mather, J. 1990, ApJ, 350, 104

Dwek, E., Foster, S. M., \& Vancura, O. 1996, ApJ, 457, 244

Eckart, A., Cameron, M., Rothermel, H., et al. 1990, ApJ, 363, 451

Fassett, C. I., \& Graham, J. A. 2000, ApJ, 538, 594

Gabriel, C., Acosta-Pulido, J., Heinrichsen, I., et al. 1997, in Astronomical Data Analysis Software and Systems VI, ed. G. Hunt, \& H. E. Payne, ASP Conf. Ser., 125, 108

Graham, J. A. 1998, ApJ, 502, 245

Haas, M., Klaas, U., Coulson, I., Thommes, E., \& Xu, C. 2000, A\&A, 356, L83

Haynes, R. F., Cannon, R. D., \& Ekers, R. D. 1983, Proc. ASA, 5, 241

Hibbard, J. E., \& van Gorkom, J. H. 1996, AJ, 111, 655

Hibbard, J. E. 2000, in Dynamics of Galaxies: from the Early Universe to the Present, ed. F. Combes, G. A. Mamon, \& V. Charmandaris, ASP Conf. Ser., 197, 285

Hibbard, J. E., van Gorkom, J. H., Rupen, M. P., \& Schiminovich, D. 2001, in Gas and Galaxy Evolution, ed. J. E. Hibbard, J. H. van Gorkom, \& M. P. Rupen, ASP Conf. Ser., 240, 657

Hildebrand, R. H. 1983, QJRAS, 24, 267

Helfer, T. T., Thornley, M. D., Regan, M. W., et al. 2003, ApJS, 145, 259

Hook, R. N., \& Fruchter, A. S. 1997, Astronomical Data Analysis Software and Systems VI, ed. G. Hunt, \& H. E. Payne, ASP Conf. Ser., 125, 147

Israel, F. P. 1998, A\&A Review, 8, 237

Karovska, M., Fabbiano, G., Nicastro, F., et al. 2002, ApJ, 577, 114

Kormendy, J. 1984, ApJ, 287, 577

Kessler, M. F., Steinz, J. A., Anderegg, M. E., et al. 1996, A\&A, 315, L27

Lemke, D., Abolins, J., Abraham, P., et al. 1996, A\&A, 315, L64 
Lemke, D., \& Klaas, U. 1999, in The Universe as seen by ISO, ed. P. Cox, \& M. F. Kessler, ESA-SP 427, 55

Leeuw, L. L., Hawarden, T. G., Matthews, H. E., Robson, E. I., \& Eckart, A. 2002, ApJ, 565, 131

LeWinter, R., Rupen, M., \& Wang, Z. 1993, BAAS, 25, 1418

Malin, D. F. 1978, Nature, 276, 591

Malin, D. F., \& Carter, D. 1980, ApJ, 274, 534

Malin, D. F., Quinn, P. J., \& Graham, J. A. 1983, ApJ, 272, L5

Marston, A. P., \& Dickens, R. J. 1988, A\&A, 193, 27

Marston, A. P. 1992, in Relationships between active galactic nuclei and starburst galaxies, ed. A. V. Filippenko, ASP Conf. Ser., 31, 123

Melo, V. P., Perez Garcia, A. M., Acosta-Pulido, J. A., Munoz-Tunon, C., \& Rodriguez Espinosa, J. M., ApJ, 574, 709

Mirabel, I. F., Laurent, O., Sanders, D. B., et al. 1999, A\&A, 341, 667

Morganti, R., Robinson, A., Fosbury, R. A. E., et al. 1991, MNRAS, 249,91

Morganti, R., Killeen, N. E. B., Ekers, R. D., \& Oosterloo, T. A. 1999, MNRAS, 307, 750

Neininger, N., Guelin, M., Ungerechts, H., Lucas, R., \& Wielebinski, R. 1998, Nature, 395, 871

Nelson, A. E., Zaritsky, D., \& Cutri, R. M. 1998, AJ, 115, 2273

Peng, E. W., Ford, H. C., Freeman, K. C., \& White, R. L. 2002, AJ, 124,3144

Petric, A., Schiminovich, D., van Gorkom, J. H., et al. 1997, BAAS, 191,8212

Regan, M. W., Thornley, M. D., Helfer, T. T., et al. 2001, ApJ, 561, 218

Rejkuba, M., Minniti, D., Silva, D. R., \& Bedding, T. R. 2001, A\&A, 379,781
Rejkuba, M., Minniti, D., Courbin, F., \& Silva, D. R. 2002, ApJ, 564, 688

Richter, O.-G., Sackett, P. D., \& Sparke, L. S. 1994, AJ, 107, 99

Ristorcelli, I., Serra, G., Lamarre, J. M., et al. 1998, ApJ, 496, 267

Ryan-Weber, E., Webster, R., \& Bekki, K. 2003, in The IGM / Galaxy Connection: The Distribution of Baryons at $z=0$, ASSL Conf. Proc. Vol. 281, ed. J. L. Rosenberg, \& M. E. Putman (Dordrecht: Kluwer), 223

Schiminovich, D., van Gorkom, J. H., van der Hulst, J. M., \& Kasow, S. 1994, ApJ, 423, L101

Schiminovich, D., van Gorkom, J. H., van der Hulst, J. M., \& Malin, D. F. 1995, ApJ, 444, L77

Schiminovich, D. 2001, in Gas and Galaxy Evolution, ed. J. E. Hibbard et al., ASP Conf. Ser., 240, 147

Sodroski, T. J., Bennett, C., Boggess, N., et al. 1994, ApJ, 428, 638

Stickel, M., Lemke, D., Klaas, U., et al. 2000, A\&A, 359, 865

Stickel, M., Bregman, J. N., Fabian, A. C., White, D. A., \& Elmegreen, D. M. 2003, A\&A, 397, 503

Sulentic, J. W., Rosado, M., Dultzin-Hacyan, D., et al. 2001, AJ, 122, 2993

Temi, P., Mathews, W. G., Brighenti, F., \& Bregman, J. D. 2003, ApJ, 585, L121

Thronson, H. A. Jr, Bally, J., \& Hacking, P. 1989, AJ, 97, 363

Tielens, A. G. G. M., McKee, C. F., Seab, C. G., \& Hollenbach, D. J. 1994, ApJ, 431, 321

Tóth, L. V., Hotzel, S., Krause, O., et al. 2000, A\&A, 364, 769

Weil, M. L., \& Hernquist, L. 1993, ApJ, 405, 142

Wilkinson, A., Browne, I. W. A., \& Wolstencroft, R. D. 1987, MNRAS, 228, 933 